\title{
Relocating Underwater Features Autonomously Using Sonar-Based SLAM
}

\author{
Maurice F. Fallon, John Folkesson, Hunter McClelland, and John J. Leonard
}

\begin{abstract}
This paper describes a system for reacquiring features of interest in a shallow-water ocean environment, using autonomous underwater vehicles (AUVs) equipped with low-cost sonar and navigation sensors. In performing mine countermeasures, it is critical to enable AUVs to navigate accurately to previously mapped objects of interest in the water column or on the seabed, for further assessment or remediation. An important aspect of the overall system design is to keep the size and cost of the reacquisition vehicle as low as possible, as it may potentially be destroyed in the reacquisition mission. This low-cost requirement prevents the use of sophisticated AUV navigation sensors, such as a Doppler velocity log (DVL) or an inertial navigation system (INS). Our system instead uses the Proviewer 900-kHz imaging sonar from Blueview Technologies, which produces forward-looking sonar (FLS) images at ranges up to $40 \mathrm{~m}$ at approximately $4 \mathrm{~Hz}$. In large volumes, it is hoped that this sensor can be manufactured at low cost. Our approach uses a novel simultaneous localization and mapping (SLAM) algorithm that detects and tracks features in the FLS images to renavigate to a previously mapped target. This feature-based navigation (FBN) system incorporates a number of recent advances in pose graph optimization algorithms for SLAM. The system has undergone extensive field testing over a period of more than four years, demonstrating the potential for the use of this new approach for feature reacquisition. In this report, we review the methodologies and components of the FBN system, describe the system's technological features, review the performance of the system in a series of extensive in-water field tests, and highlight issues for future research.
\end{abstract}

Index Terms-Marine navigation, marine vehicles, mobile robots, sensor fusion, simultaneous localization and mapping (SLAM), sonar detection, synthetic aperture sonar, underwater technology.

\section{INTRODUCTION}

$\mathbf{N}$ UMEROUS scientific, military, and commercial applications of autonomous underwater vehicles (AUVs) can benefit from the capability to renavigate to objects of interest that have been mapped during a previous mapping

Manuscript received October 10, 2011; revised June 04, 2012; accepted December 14, 2012. Date of publication April 26, 2013; date of current version July 10, 2013. This work was supported by the U.S. Office of Naval Research under Grants N00014-05-1-0244 and N00014-11-10119.

Guest Editor: M. Seto

M. F. Fallon and J. J. Leonard are with the Computer Science and Artificial Intelligence Laboratory, Massachusetts Institute of Technology (MIT), Cambridge, MA 02139 USA (e-mail: mfallon@mit.edu; jleonard@mit.edu).

J. Folkesson is with the Center for Autonomous Systems, Kungliga Tekniska Högskolan (KTH), Stockholm 100 44, Sweden (e-mail: johnf@kth.se).

$\mathrm{H}$. McClelland is with the Robotics and Mechanisms Laboratory, Virginia Polytechnic Institute of Technology and State University (Virginia Tech), Blacksburg, VA 24061 USA (e-mail: hgm@vt.edu).

Color versions of one or more of the figures in this paper are available online at http://ieeexplore.ieee.org.

Digital Object Identifier 10.1109/JOE.2012.2235664 session. One such application is the neutralization of mines in shallow water - a task that has traditionally been carried out by human divers. The potential for casualties has motivated the development of unmanned systems to replace human divers. While a tethered robotic vehicle could be remotely controlled to perform mine countermeasures (MCMs), a solution using untethered AUVs offers numerous mobility and independence advantages. Our goal for this work is to enable small, low-cost AUVs to robustly reacquire previously mapped features of interest, whether floating in the water column or lying on the seabed.

When mission requirements dictate that vehicle cost must be extremely low, the navigation problem for feature reacquisition is quite challenging. The primary challenge is to achieve good navigation performance using only very low-cost sensors. To do this, we propose to use an a priori map, built by other vehicles with more capable (and expensive) sensing as input to our feature-based navigation (FBN) software running on the low-cost reacquisition vehicle.

The ocean environment places severe challenges on the operation of AUVs. Communication with an untethered AUV via acoustic modems is typically restricted to low rates (bytes per second) and with several seconds of latency, such that interaction with the AUV is primary restricted to command and control of the vehicle [1]. Furthermore, due to the scattering and absorption effects of water, typical terrestrial sensors such as LIght Detection and Ranging (LIDARs) and cameras are not suitable for use in many environments of interest. Finally, operational conditions in littoral waters are often challenging with high tides, wind-driven currents, and wave surge, which all heavily affect the robot's position estimation as well as increasing operator risk during field operations. Access to the Global Positioning System (GPS) is not possible underwater and acoustic long-baseline transponders (and related technologies) can drift or be lost during the time lapse between deployment and use in a mission.

Nevertheless, unmanned underwater vehicles deployed with expensive inertial sensors, such as Doppler velocity loggers (DVLs) and fiber optic gyroscopes (FOGs) can successfully negotiate complex underwater terrains [2], [3]. Sophisticated instruments of this type would enable an AUV to navigate to and relocalize a mine, however the neutralization would be prohibitively expensive. Less costly (and, therefore, expendable) AUVs, guided with only onboard inertial sensors, would typically encounter performance problems that would render them inefficacious in carrying out MCM.

Therefore, we have developed an approach based on the use of low-cost forward-looking sonar (FLS) technology to estimate 
vehicle motion as well as the location of features of interest so as to reach the a priori designated feature of interest (FOI). In this paper, we describe the components of the system including FLS feature detection and tracking, local map estimation, global map matching, and terminal homing. The structure of the remainder of this document is as follows. Section II provides an overview of the FBN system for target reacquisition. Section III describes the Proviewer FLS and provides a detailed overview and performance analysis of the sonar processing tool chain we have developed. Section IV discusses the SLAM engine at the heart of our AUV's navigation system before explaining how this map can be matched to an a priori map in Section V. Section VI presents some examples of the performance of our system in increasingly difficult operating scenarios. Finally, Section VII concludes this paper with a summary of our contributions and a discussion of issues warranting future research.

\section{SySTEM OVERVIEW}

Our mission scenario envisages revisiting a FOI located on (or slightly above) a seabed and surrounded by significant clutter. To aid in this task the feature field is first uniformly surveyed by a high-quality AUV [such as the REMUS 100 in Fig. 1(c)] that navigates using acoustic beacons or an inertial navigation system (INS) with a DVL to acquire accurate sidescan sonar data of the region of interest. Using the sidescan sonar data, a mosaic can be generated, as illustrated in Fig. 2. Human operators then analyze this imagery to extract an $a$ priori map of features as well as identifying the FOI. The accuracy of the feature locations in this map is typically $5 \mathrm{~m}$. These features may be man-made or natural objects on the seafloor as well as moored objects in the water column and represent the "local universe" of the FOI. The features varied greatly in size and shape, and were typically less than $1 \mathrm{~m}$ in diameter.

This map and the location of the FOI acts as input to a second low-cost relocalization vehicle. In the mission scenario, we aim to release this vehicle at a distance of 100-1500 $\mathrm{m}$ from the center of the prior map and have it swim at the surface close to the feature field before diving to the seabed. Upon reentering this feature field, the vehicle will extract features from its sonar imagery and use these features to build a map, while concurrently estimating its position. This process is commonly known as simultaneous localization and mapping (SLAM) [4].

Having reobserved a sufficient number of features, the AUV will localize relative to the a priori map and then home to the FOI. If successful, the AUV will self-detonate or place an explosive charge. Because of this, the vehicle is not intended to be recoverable. For these reasons, a low-cost vehicle design requirement has had significant impact on the SLAM algorithms mentioned here. More detail about the reattachment process can be found in Section V-C.

Test runs of these reacquisition operations have been conducted over several years on three different vehicles, shown in Fig. 1: an iRobot Ranger, an iRobot Transphibian; and a Hydroid REMUS 100.

\section{A. Overview of Vehicles Used}

The primary platform used during development has been the iRobot Ranger AUV [5]. This vehicle was equipped with a depth

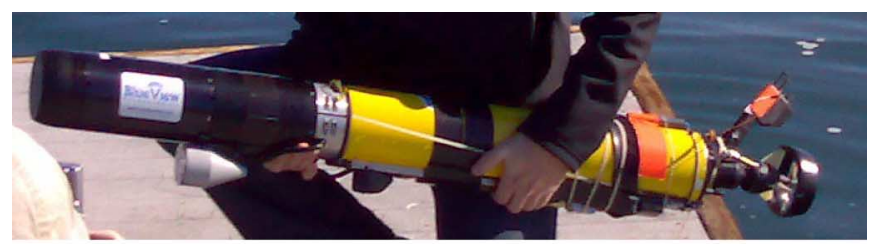

(a)

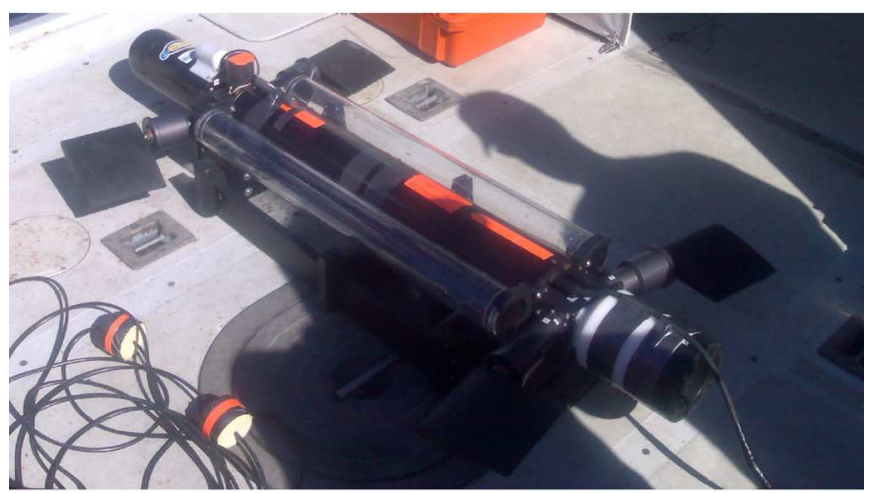

(b)

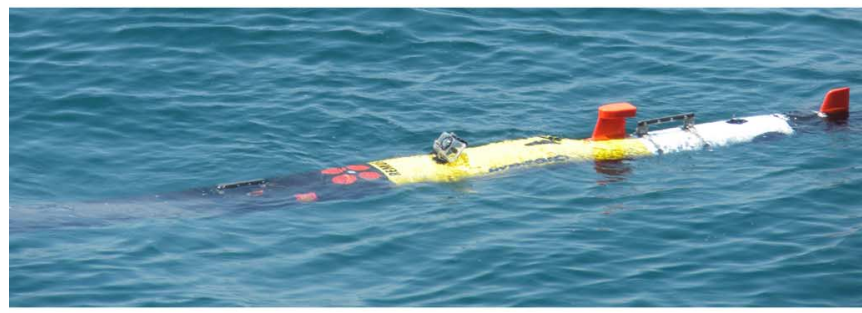

(c)

Fig. 1. The AUVs used in the project: (a) iRobot Ranger-a low-cost single-man portable AUV; (b) iRobot Transphibian equipped with fin actuators to allow for six-degree-of-freedom maneuverability; and (c) Hydroid REMUS 100 used primarily as the survey vehicle.

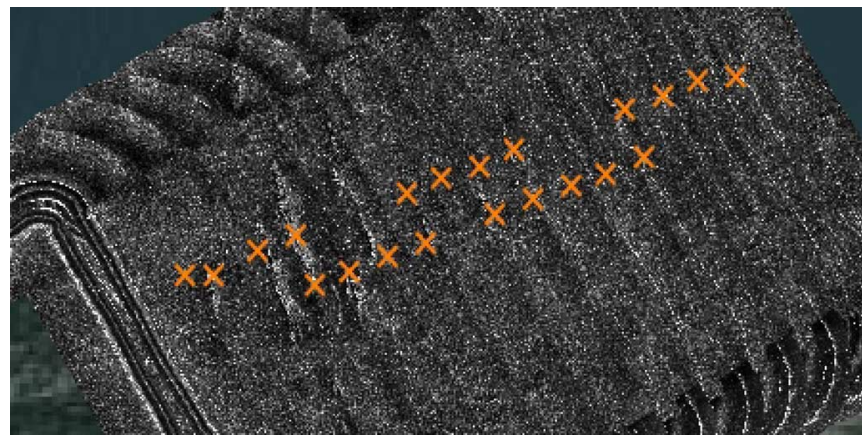

Fig. 2. A typical prior map generated using a REMUS 100 equipped with a MarineSonics sidescan sonar. A series of features were extracted by trained human operators from the sidescan sonar imagery to produce this map for the target reacquisition mission. The distance between the features is approximately $20 \mathrm{~m}$. Figure courtesy of SeeByte Ltd.

sensor, an altimeter, a GPS receiver, a 3-D compass, an acoustic pinger, and a Blueview Proviewer 900-kHz FLS. The vehicle's design was intended to be low cost and lightweight. As indicated by Fig. 1(a), it is single-man portable and deployable.

The design of the vehicle incorporates a servoed propeller, which allows the vehicle to be highly maneuverable with a tight turning radius of $0.5 \mathrm{~m}$. This compares to $10 \mathrm{~m}$ for the REMUS 100. This is of particular importance for the target homing at the end of the mission (see Section V-C) as a vehicle with a 
lower turning radius can more reliably seek to its intended target without needing to come about (and potentially loose track of the target).

Since dead reckoning is the prime source of error growth, minimizing the distance to turn-and-capture increased the chance of a successful mission. The cruising speed of the AUV is quite low at about $0.6 \mathrm{~m} / \mathrm{s}$, comparable with typical ocean surface currents. Thus, the dead-reckoning error due to the current can be quite significant. For that reason, we developed a method to estimate the ocean current online. Our approach is discussed in Section II-B.

The vehicle specifically did not have a DVL, used for precise velocity estimation, due to cost limitations. Currently available DVLs cost approximately $\$ 20000$. It would be remiss of us not to mention that the current range of FLS devices are comparably expensive, however the manufacturer expects that mass production can reduce cost by an order of magnitude, as the current price is related to the initial cost of research and development. Nonetheless, the utility of the capabilities outlined herein go far beyond this particular application.

Additionally, underwater vehicles typically use industrial processor designs that have more limited processing capability than typical desktop computers. This places severe computing restrictions on the operating vehicle, which we will discuss in Section III.

Other Vehicles: While most of our testing focused on the Ranger AUV, some experiments were also carried out with a biologically inspired AUV - the Transphibian [6] — which is equipped with fin actuators to allow for six-degree-of-freedom maneuverability and a variable buoyancy engine allowing for complete servoing around the FOI. Finally, while the Hydroid REMUS 100 was primarily used as a survey vehicle (as discussed in Section IV), it has also been used in the experiments to evaluate some sonar detector benchmarks in Section III-B. Doing this also demonstrates the generality of the algorithmic solution we have proposed.

\section{B. Vehicle Dead Reckoning and Current Estimation}

At the core of our SLAM system is a dead-reckoning algorithm similar to others presented in the literature. High-frequency depth estimates, altimeter altitudes, GPS fixes, and compass estimates of roll, pitch, and heading are fused with actuation values (orientation of the servoed propeller and the estimated propeller rotation speed) by propagating a typical extended Kalman filter (EKF) to produce an estimate of the vehicle position and uncertainty. Consider [7] for a full overview of the state of the art. In benign current-free conditions, with careful tuning and excellent compass calibration, this procedure produced a dead-reckoning estimate with about $1 \%-2 \%$ error per distance traveled. However, as we transitioned to more realistic test locations, a more typical error was of the order of $3 \%$. By comparison, FOG-based navigation systems can achieve drift rates below $0.1 \%$ in the open ocean. See [8] for a list of such systems.

In current-prone conditions in later stages of the project (as discussed in Section VI) a current estimation model was developed so as to reject the vehicle's drift in this situation. (Because of the nature of this project, it was not possible to use the aforementioned DVL-enabled vehicle's estimate of the current profile.) Immediately prior to a mission, as the AUV approaches the feature field, it was programmed to carry out a series of dives of increasing depth (between three and five in total). Between each dive, it surfaced and used GPS fixes to compare its actual position to the position estimated without factoring in current modeling, and then used those comparisons to compute the ocean current estimate. No acoustic Doppler current profiler (ADCP) was present on the vehicle.

Estimating the current profile in this manner is problematic for a number of reasons-primarily because the parameters at greater depths are estimated using those at shallower depths. Additionally, the following issues are not and cannot be modeled using our sensor suite:

1) momentary surge currents;

2) currents rising and falling in the horizontal water column;

3) current profiles with numerous layers moving in different directions at unknown depths;

4) foreign objects altering the vehicle dynamics (such as seaweed entangling the propeller).

Aware of these limitations, we assumed a two-current model: a surface current $(\vec{S}$, primarily due to wind) and a deeper laminar current $(\vec{D}$, due to the tide). These currents are estimated as 2-D vectors in the horizontal plane.

At depths less than $0.5 \mathrm{~m}$, the surface current is assumed to be dominant. Below this there is a transition between the two currents. Finally, at the seabed, the laminar current decays to zero-fitted as a square root to the vehicle altitude. Numerically, the combined current vector at a specific depth is

$$
\vec{C}(a, d)=\sqrt{a /(a+d)}(w(d) \vec{S}+\vec{D})
$$

where $a$ is the AUV altitude, $d$ is the AUV depth, and $w(d)$ is the depth weighting of the surface current and was calculated as follows:

$$
w(d)= \begin{cases}1, & \text { for } d \leq d_{0} \\ 1-k\left(d-d_{0}\right) /|\vec{S}|, & d_{0}<d \text { and } d \leq|\vec{S}| / k+d_{0} \\ 0, & \text { otherwise }\end{cases}
$$

where $d_{0}=0.5 \mathrm{~m}$, representing the boundary between the surface region and the rest of the water column, and $k=0.2$ was empirically fixed to degrade this effect with increased depth.

This simplistic model performed reasonably well in smaller currents (below $0.3 \mathrm{~m} / \mathrm{s}$ ) and allowed the AUV to locate the feature field after ingressing using solely dead reckoning. After entering, the field success was primarily due to the sonar-based SLAM algorithm (outlined in Section IV). At $0.3 \mathrm{~m} / \mathrm{s}$, we were able to enter the field approximately $85 \%$ of the time using this model, and we estimate the error as about $5 \%$ per distance traveled.

However, when operating in high currents (close to or greater than the velocity of the vehicle), this model was not sophisticated enough to produce a sufficiently accurate position estimate for the AUV to find the feature field. While the vehicle could surface to reacquire GPS and then try again, the oceancurrent-to-maximum-AUV-speed ratio of $50 \%-70 \%$ represents 

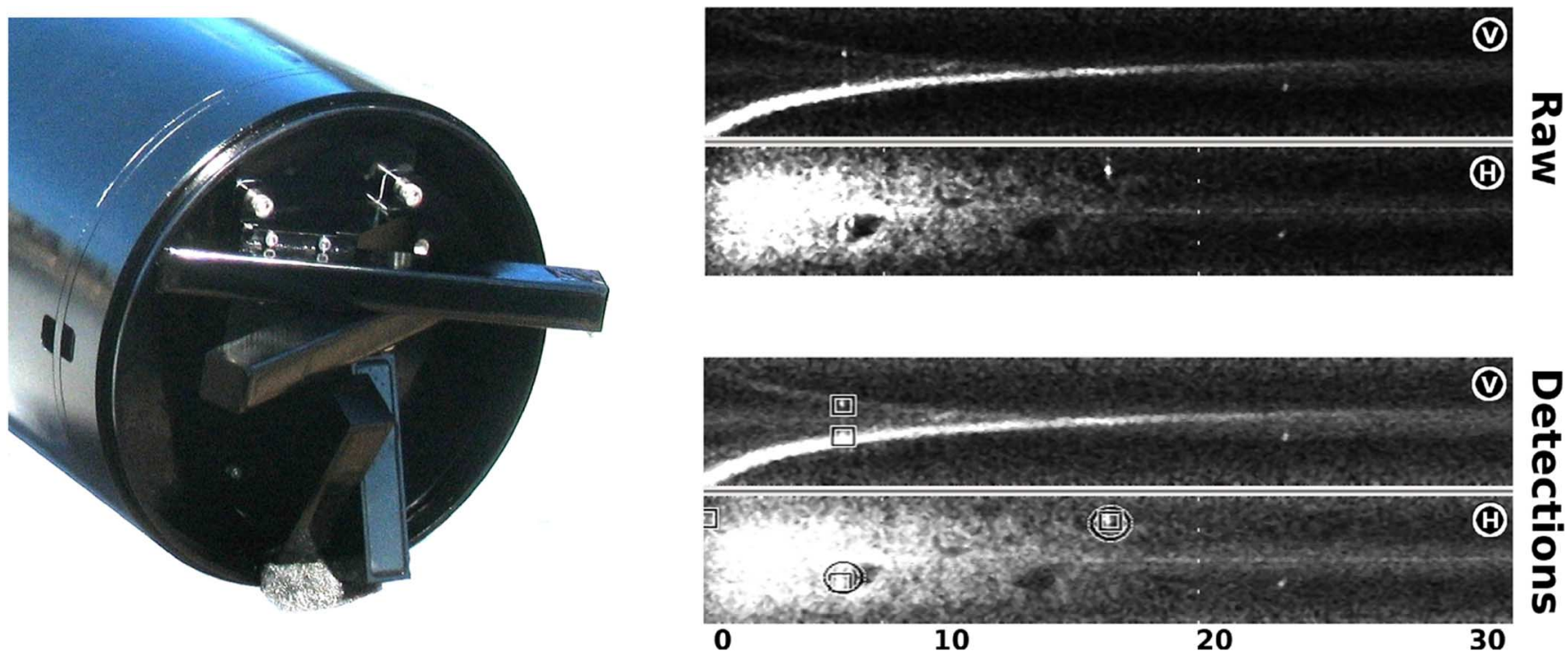

Fig. 3. This $45^{\circ}$ blazed array sonar consists of two perpendicular heads, each made up of a pair of $22^{\circ}$ sonar transducers (left). Each head produces a raw image, the upper pair (right), which we then automatically process to detect the features marked in the lower pair (right). In each case, the vertical image is above the horizontal image. Both images are presented in polar coordinates with increasing ranges to the right, and right-to-left angle going vertically up. Note that the distinctive line in the vertical image is the observation of the seabed (which appears curved due to the use of polar coordinates here).

a limitation to this or any other AUV perception system. This limitation is further discussed in Section VII.

As this system was developed in the absence of independent measurements of the currents at different altitudes, we refer the interested reader to a more fundamental treatment of ocean dynamics such as that presented in [9]. Given the limitations of our approach, a more accurately guided AUV is likely to have greater success in these challenging current regimes.

\section{Proviewer FLS Processing}

The sonar is our most important sensor: enabling the AUV to observe the features in its environment and to act based on these observations. During the project, a series of Blueview Proviewer FLS sonars were used. In this section, we give an overview of the sensor technology before presenting our sonar processing algorithms in Section III-A.

The Proviewer FLS operates using blazed array technology [10]. Typically, the sonar consisted of two heads (horizontal and vertical) each with a field of view (FOV) of $45^{\circ}$. Units with $90^{\circ}$ and $135^{\circ} \mathrm{FOV}$ were later used and the statistics presented in Section III-B were calculated using the $90^{\circ}$ version. Each head is made up of individual $22.5^{\circ}$ sonar transducers, as illustrated in Fig. 3. Additionally, the horizontal transducers were tilted down $5^{\circ}$ to best image the seabed as the vehicle typically flew horizontally and about $2 \mathrm{~m}$ above the sea bottom.

The sensor works by transmitting an ensonifying signal (colloquially known as a "ping"), which reflects off of objects of incidence (in particular, metal and rock) and is received by the transducer. The phase, amplitude, and delay of the returned signals are processed to produce the pattern indicated in Fig. 4. This return is evaluated for each array element with $1^{\circ}$ resolution in the plane of the head, and the output is then fused together by Blueview's onboard software to produce the image in Fig. 5.

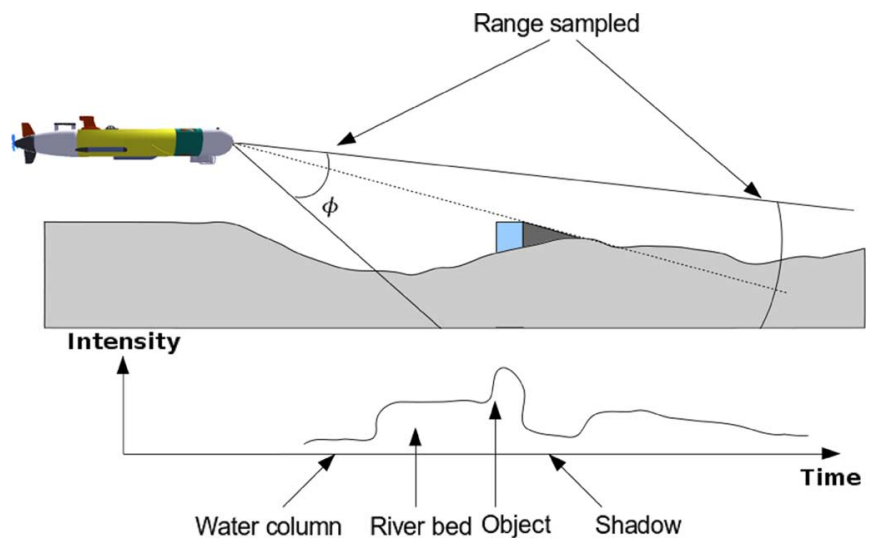

Fig. 4. This figure illustrates the sonar image generation process for a single sonar beam. Each beam return is a vector of intensities of the returned sonar signal with objects of high density resulting in high returns and shadows formed by lower intensities.

Sonar image formation is dramatically different from regular optical image formation [11]. Due to the reflected ensonification, the primary visual cue of the location of a feature is often not at its location, but rather beyond it, as indicated by a shadow of intensity values below the noise floor, in Fig. 4. This, coupled with some increased amplitude and a gradient at the feature location, are the primary cues used in Section III-A to extract features. The length of the shadow can be indicative of the height of the feature, although moored features result in features detached from their shadows.

The outgoing sonar signal also has a significant lobe width, $\phi \sim 20^{\circ}$, which means that there is significant ambiguity as to the location of the object in the axis normal to the sensed plane. In Section IV-B and Fig. 9, we explain how we fused these observations so as to estimate the elevation of detections using only the horizontal image. 

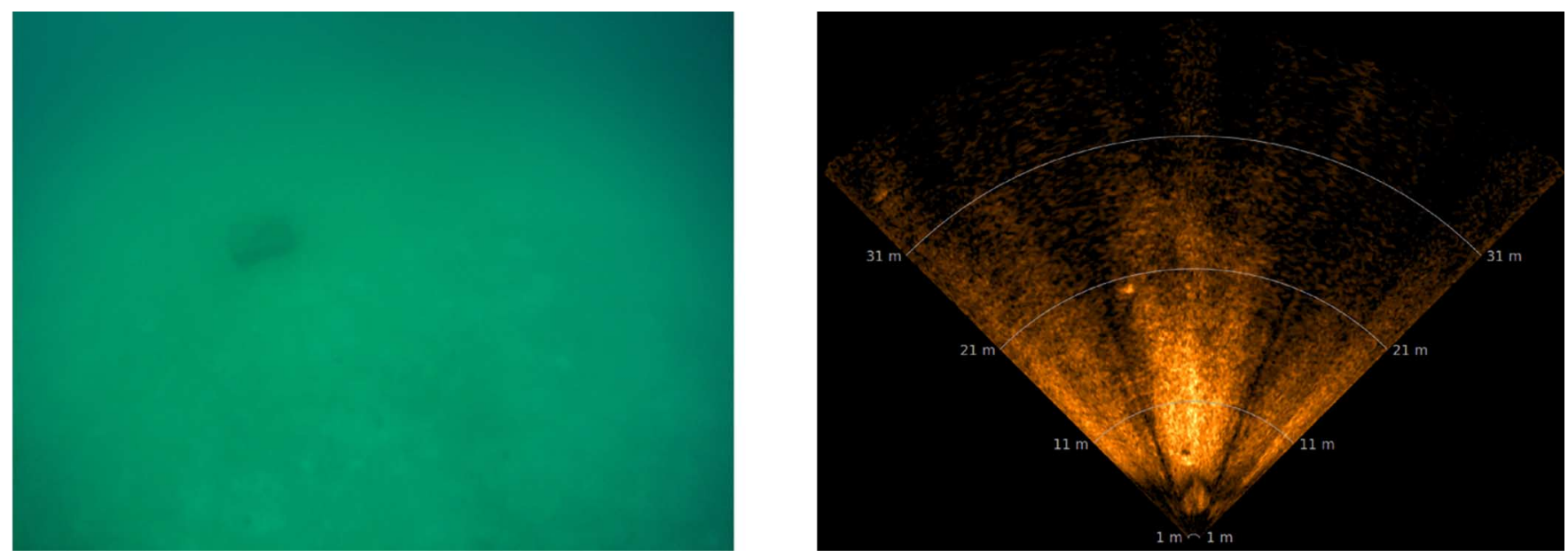

Fig. 5. Typical underwater camera and sonar images (approximately synchronized). The clear water and well-lit scenario represents some of the best possible optical conditions, nonetheless visibility is only a few meters. The $90^{\circ}$ blazed array sonar horizontal image indicates three features (one at $5 \mathrm{~m}$ in front; one at 20 $\mathrm{m}$ and $5^{\circ}$ to the left; and one at $35 \mathrm{~m}$ and $40^{\circ}$ to the left), which is more than typical.

\section{A. Sonar Feature Detection}

In this section, we outline our algorithms that extract point features from regions of high contrast. Williams et al. [12] implemented a similar processing pipeline to ours: beginning with raw data and progressively processing it to extract point features which are then tracked to carry out SLAM. Related previous work in our laboratory [13] also implemented a similar tool chain, but was focused on dense registration rather than explicit point feature detection and extraction.

In related work, Ruiz et al. [14] performed online sidescanbased SLAM, but, as they suggest, this approach is not suitable for single pass operations such as the proposed mission. Fairfield et al. [15] utilized pencil-beam sonars to map flooded tunnels and sink holes. Using a particle filter, this approach can avoid explicit data association but the environment considered is quite different from the open ocean. Meanwhile, FLS has also been used for obstacle detection and path planning [16]. In this application, the feature extraction is focused on conservative estimation of all detected objects given the noisy output of the FLS systems. Finally, Clark and Bell [17] describe multitarget tracking of multiple features from an FLS using a probability hypothesis density (PHD) filter.

The processing tool chain is carried out on the polar coordinate image produced by the Blueview SDK (Fig. 3) rather than the Cartesian image (Fig. 5). The maximum range of the Blueview FLS is $40 \mathrm{~m}$, and images are typically processed at $3-4 \mathrm{~Hz}$. Objects were typically visible up to $20 \mathrm{~m}$ away, while brightly reflective objects are detectable at $40 \mathrm{~m}$.

Given the performance requirements of the platform, the algorithm is required to be adaptive to bottom-reflective brightness. This is achieved by the online estimation of an average background image immediately after the vehicle levels out at its cruising depth. Estimating this background noise image, which is highly dependent on pitch and altitude as well the seabed material, is essential to achieve excellent performance in both sandy and muddy bottomed environments.
The steps used to process the images are as follows:

1) form a $50 \times 157$ image of the vertical head sonar data (as in Fig. 3);

2) find sea bottom, altitude, and relative slope using line extraction;

3) if slope (i.e., vehicle pitch) is too far from level flight, stop processing;

4) form a $50 \times 157$ image of the horizontal head sonar data;

5) using the altitude (from step 2), select an altitude-specific averaged background image;

6) lowpass filter the current horizontal image into the background image;

7) subtract the background image from the horizontal image to form the shadow image;

8) segment vertical image into bottom and open water regions;

9) segment horizontal image into three regions: a) near-field open water, b) ranges where the bottom is sufficiently bright to see shadows, and c) the remaining range out to $40 \mathrm{~m}$;

10) lowpass filter along each bearing to eliminate very small features and noise;

11) search for edges as gradients in each segment with thresholds based on the average background image level (this gives some adaption to different bottom types);

12) search for a feature indicated by a bright area close to the sensor followed by a shadow (below the noise floor);

13) select for output any features found in both heads as well as the strongest remaining detections in each segment.

This relatively simple detection algorithm is implemented using integer arithmetic and optimized for speed. This tool chain is similar to that presented previously in [13], including the figures presented therein.

In terms of computing load, the feature detector uses little processing power. The formation of the input image (using the Blueview SDK), the input to this process, requires a substantial $180 \mathrm{~ms}$ per image. The feature detector requires about $18 \mathrm{~ms}$, 


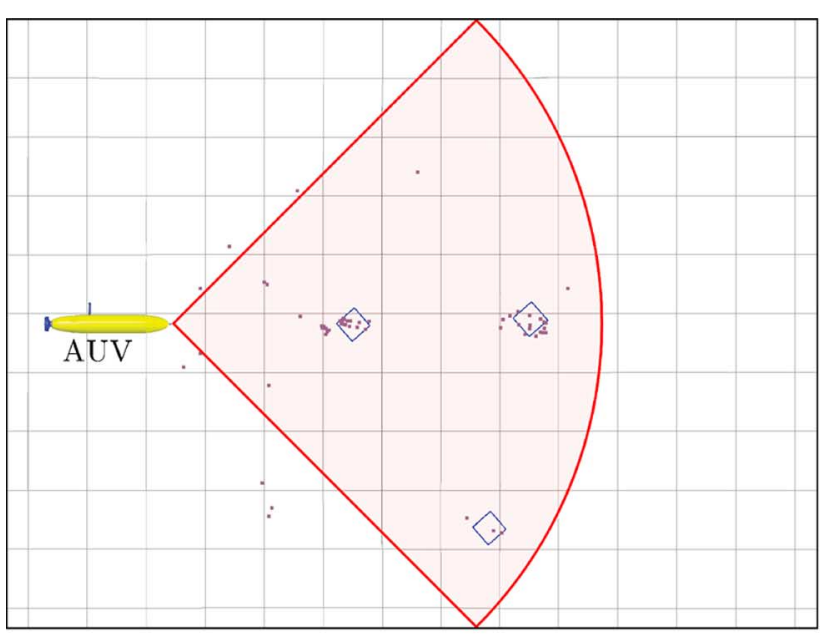

(a)

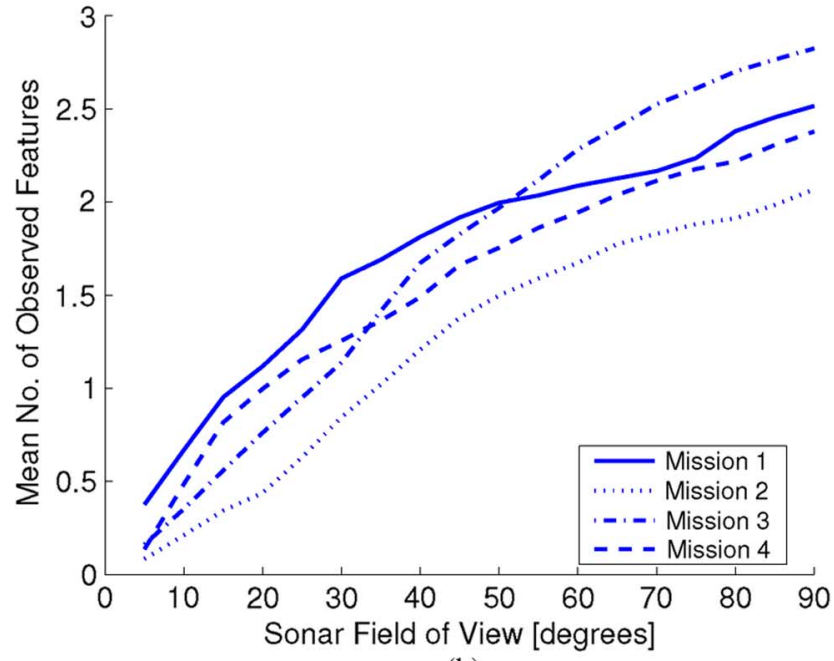

(b)

Fig. 6. (a) Typical feature detection performance. The AUV is on the left-hand side, and its $90^{\circ}$ sonar footprint is indicated by the red wedge. Each purple dot indicates a sonar point feature extraction. Two features at the center of the footprint have been detected several times. The third feature located at the side of the sonar footprint would not have been recognized using a $45^{\circ} \mathrm{FOV}$ sonar. The scale of the grid is $5 \mathrm{~m}$ and the maximum range $40 \mathrm{~m}$. (b) Analysis of the average number of features detected at any instance during our missions, while varying the sonar FOV.

while the remaining central processing unit (CPU) power is used to fuse the measurements, to make high level mission decisions, and to control the AUV.

Ongoing work by the manufacturer to reduce this time (by forming the sonar image on the device) would allow a great increase in the processing frequency, which, in turn, will improve the tracking and mapping performance.

\section{B. Sonar Processing Performance Evaluation}

To benchmark the performance of the feature detector, we present a series of experiments, in which a $90^{\circ}$ FLS was attached to the FOG-enabled REMUS 100 in Fig. 1(c). Patterns similar to the missions outlined in Section VI were carried out. Because of the shortness of the missions (10-15 min) and quality of navigation, the AUV accumulated negligible positioning error when compared to the Ranger platform, and, in what follows, we assume its position estimate to be the "ground truth" position.

The vehicle navigated a field of features arranged as in Fig. 2 whose prior locations were accurate to about $5 \mathrm{~m}$. Four different missions on the same feature field were carried out from different directions and along different approaches. A total of 17 features were in the field: a mixture of natural and man-made, both moored and lying on the seabed. Time spent within the feature field was typically $180 \mathrm{~s}$, during which about 300 sonar pings were detected. The vehicle traveled at approximately 1.3 $\mathrm{m} / \mathrm{s}$. Typical feature detection performance is shown in Fig. 6 .

1) Precision: The first metric we will consider, precision, is the ratio of correctly detected features to the total number of extracted features. We define a correctly detected feature as one which lies within a certain distance $r_{\text {thres }}$ of a point feature $p_{i}$ from the a priori map

$$
C_{i}= \begin{cases}1, & \text { if }\left\|d_{i}-p_{j}\right\| \leq r_{\text {thres }} \forall j \\ 0, & \text { otherwise }\end{cases}
$$

where $\left\|d_{i}-p_{j}\right\|$ represents the Euclidean distance between the two entities - evaluated in 2-D for simplicity. The detection ratio is then simply

$$
D=\frac{\sum_{i=1}^{F} C_{i}}{F}
$$

where $F$ is the total number of features detected. $D$ lies in the range $0 \leq D \leq 1$.

Results are presented in Fig. 7. In summary, approximately $70 \%$ of detections fall within $3 \mathrm{~m}$ of a prior map feature location. The remaining $30 \%$, falsely detected noise were uniformly distributed in the rest of the sonar sensor footprint. Mission 2 exhibited significantly more turning motion; we believe that timing latency is to blame for the performance reduction in this case.

2) Recall: A second metric is that of recall-how frequently is a particular feature $f$ detected when the sonar senses the area in which it is located. To measure this parameter, we count the number of times that a feature is detected within $5 \mathrm{~m}$ of a prior map feature $D_{f}$ and express it as a fraction of the total number of times that the sonar sensed the location of the prior map feature $N_{f}$, which is simply

$$
R_{f}=\frac{D_{f}}{N_{f}}
$$

If $R_{f}=1$, then each time it was possible to sense feature $f$, it was detected. Meanwhile, $R_{f}=0$ means the feature could not be detected at all (probably because it was indistinct, obscured, or perhaps a mistake in the prior mapping process). This metric is presented in Fig. 7 for the same four missions.

For each mission, a dot represents the recall rate of a particular prior map feature, while the bar indicates the median recall rate across all features. In the case of Mission 1, on average, we detected each feature in two of every three scans that sensed the area in which it was located. 


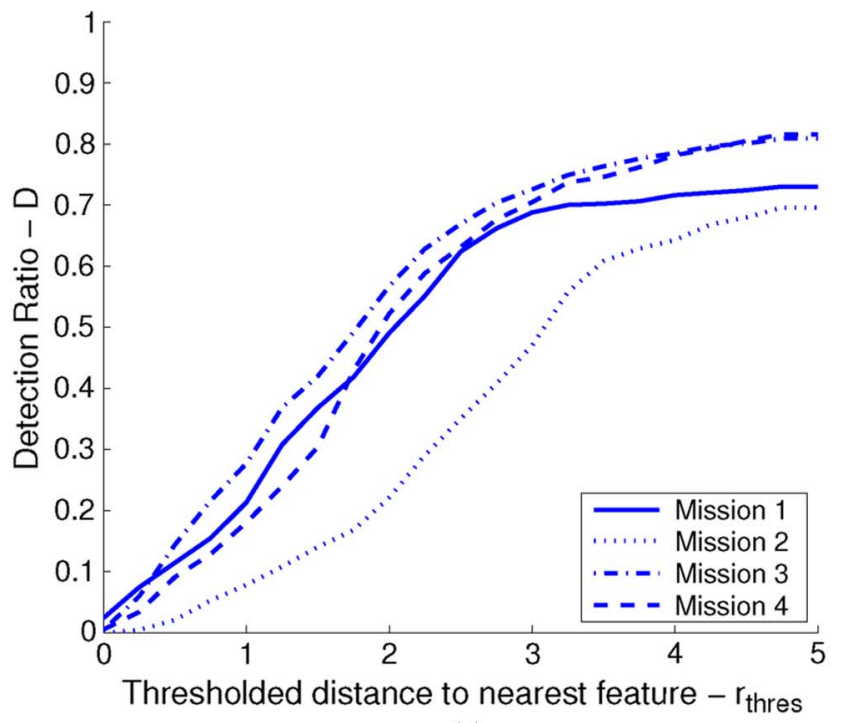

(a)

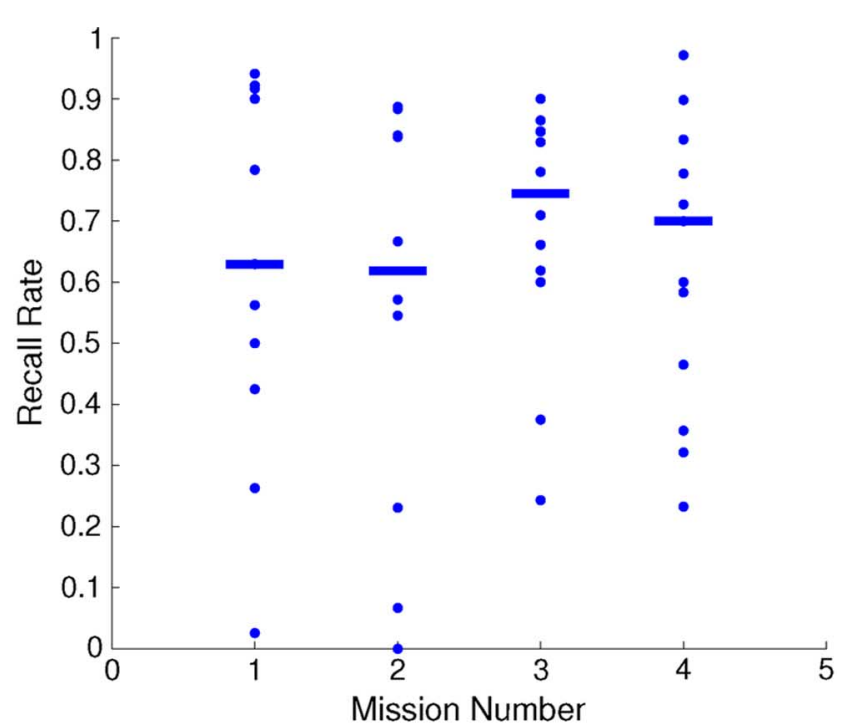

(b)

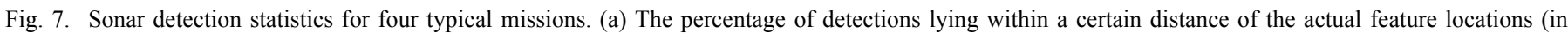

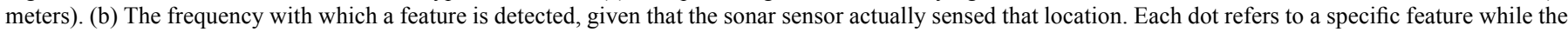
bar represents the median. See Section III-B for more detail.

3) Field of View: Using the detections from the above experiments, we also considered the effect of artificially varying the FOV of the sonar. We evaluated which feature extractions would have occurred for FOVs smaller than the $90^{\circ}$ actually used.

In the case of a $45^{\circ}$ sonar, the results show that, on average, 1.5 features would have been visible at any one time during these missions. This figure rose to 2.5 features for the $90^{\circ}$ sonar. This gives an indication of the sparsity of information available to the navigation algorithm. In particular, for perfect data association, it was typically not possible to uniquely determine the AUV's position using only a $45^{\circ}$ sensor.

Due to the mechanical design of the sonar transducers and the small diameter of the Ranger AUV, all experiments in Section VI were run with a $45^{\circ}$ sonar. Given this low aperture size, the sampling frequency, and the vehicle velocity, the AUV typically had to pass within $5 \mathrm{~m}$ of a bottom feature so as to observe it frequently enough to reliably detect it.

While these results illustrate encouraging performance of the sonar detector, inaccurate dead reckoning and a sparse feature field (often with one or no features visible at any particular time) mean that using feature detections alone to infer the vehicle's location in not possible. In Section IV, we discuss how the output of the detector is fused with the AUV navigation to jointly estimate its location and the relative position of the features.

\section{MARINE MAPPING AND LOCALIZATION}

SLAM is a core skill of any intelligent autonomous robot: jointly estimating its position and the world around it as it actively explores. The SLAM field has developed a rich literature, including EKF-based approaches such as [18] and [19], and particle filter approaches, including FastSLAM and GMapping [20], [21]. The former approaches suffer from a loss of information due to the linearization decision inherent in the EKF correction step [22]. Although the particle filter approaches solve this problem through Monte Carlo sampling, the loss of diversity during a loop closure means that, for any large-sized exploration, a prohibitively large number of particles is required.

The state of the art has emerged to be nonlinear optimization of the graph of the vehicle trajectory and feature observations. In this approach, the linearization points are regularly reevaluated allowing us to maintain a consistent solution, which can be efficiently solved online. Our implementation uses an approach similar to the square root smoother [23], [24].

The joint probability distribution of the vehicle trajectory $X=\left[\begin{array}{llll}x_{1} & x_{2} & \ldots & x_{N}\end{array}\right]$, the sonar feature detections $Z=\left[\begin{array}{llll}z_{1} & z_{2} & \ldots & z_{M}\end{array}\right]$, and the dead-reckoning measurements between successive poses $U=\left[\begin{array}{llll}u_{1} & u_{2} & \ldots & u_{N}\end{array}\right]$ are given by

$$
P(X, U, Z)=P\left(x_{0}\right) \prod_{i=1}^{N} P\left(x_{i} \mid x_{i-1}, u_{i}\right) \prod_{m=1}^{M} P\left(z_{m} \mid x_{m}, s_{m}\right)
$$

where $x_{m}$ represents the vehicle pose while observing the sonar feature $s_{m}$ at a relative position $z_{m} . P\left(x_{0}\right)$ is a prior on the initial pose.

The maximum a posteriori (MAP) estimate of the vehicle trajectory can then be formed given the measurements. Denoting this estimate $\hat{X}$, the resultant MAP estimator is given by

$$
\begin{aligned}
\hat{X} & =\underset{X}{\arg \max } P(U, Z \mid X) P(X) \\
& =\underset{X}{\arg \max } P(X, U, Z) \\
& =\underset{X}{\arg \min }-\log P(X, U, Z) .
\end{aligned}
$$

Our solution, originally described in [25], incrementally solves this nonlinear least square (NLS) optimization with some considerations for the marine environment and our limited processing capability. We start by initializing the graph with the GPS position of the AUV at dive and then add a new node to the graph representing each incremental change in AUV pose (which is synchronized to a sonar ping). 
The dead-reckoning and sonar measurements are added to the measurement matrix $A_{i}$ (at time $i$ ), which corresponds to the square root of the information matrix $H_{i}$. The information matrix is in turn the inverse of the covariance matrix for the measurement $\Sigma_{i}$

$$
A_{i}^{T} A_{i}=H_{i}=\Sigma_{i}^{-1} .
$$

Solving (7) is equivalent to minimizing the cost of the NLS optimization

$$
E=\sum_{i}\left|\left(A_{i} \triangle \mathbf{x}-\mathbf{b}_{i}\right)^{T}\left(A_{i} \triangle \mathbf{x}-\mathbf{b}_{i}\right)\right| .
$$

With each additional measurement $i$, this cost grows as

$$
\triangle E_{i}=\left(A_{i} \triangle \mathbf{x}-\mathbf{b}_{i}\right)^{T}\left(A_{i} \triangle \mathbf{x}-\mathbf{b}_{i}\right)
$$

where $\Delta \mathbf{x}$ is the change in state from the current estimated state (where $A_{i}$ and $\mathbf{b}_{i}$ are evaluated). $\mathbf{b}_{i}$ is the measurement mean and the $A_{i}$ matrix characterizes the measurement covariance. This $\triangle E_{i}$ is added to all the previous terms and the resulting sum minimized by adjusting the state

$$
\sum_{j}\left(A_{j}^{T} \sum_{i}\left(A_{i} \triangle \mathbf{x}-\mathbf{b}_{i}\right)\right)=A^{T}(A \triangle \mathbf{x}-\mathbf{b})=0 .
$$

Matrix $A$ is incrementally formed by stacking $A_{i}$ while $\mathbf{b}$ is the state vector formed by stacking $\mathbf{b}_{i}$. As a result, $A$ has rows for each measurement component and columns for each state component.

The measurement matrix can then be decomposed as $A=$ $Q R$ with $R$ upper-triangular Cholesky triangle and $Q$ an orthonormal matrix. The minimum cost state is then found by back substitution

$$
R^{T}\left(R \triangle \mathbf{x}-Q^{T} \mathbf{b}\right)=\left(R \triangle \mathbf{x}-\mathbf{b}^{\prime}\right)=0
$$

where $\mathbf{b}^{\prime}=Q^{T} \mathbf{b}$. Upon adding a new $A_{i}$, we can append it to the $R$ matrix, and the subsequent decomposition step involves only the last few states.

The complexity of this system of equations is tied to the sparseness of the $A$ matrix, which is itself dependent on the fill-in caused by loops in the graph structure. We explicitly avoid carrying out loop closures in this filter so as to maintain sparsity. All of this ensures that the matrices remain sparse and computation complexity predictable. Decomposition will not grow in complexity at each iteration while the computational cost of back substitution will grow, but it is linear.

\section{A. Composite Measurements}

So as to avoid computational growth due to an ever increasing graph size and to produce an input to the map matching stage, we periodically rationalize the oldest measurements from this graph to form a composite measurement. To do this, we marginalize out all the poses that have occurred during the previous (approximately) $10 \mathrm{~s}$ period to produce a single node for the relative motion for that period as well as nodes for fully detected features and the associated covariances. This approach is sim-

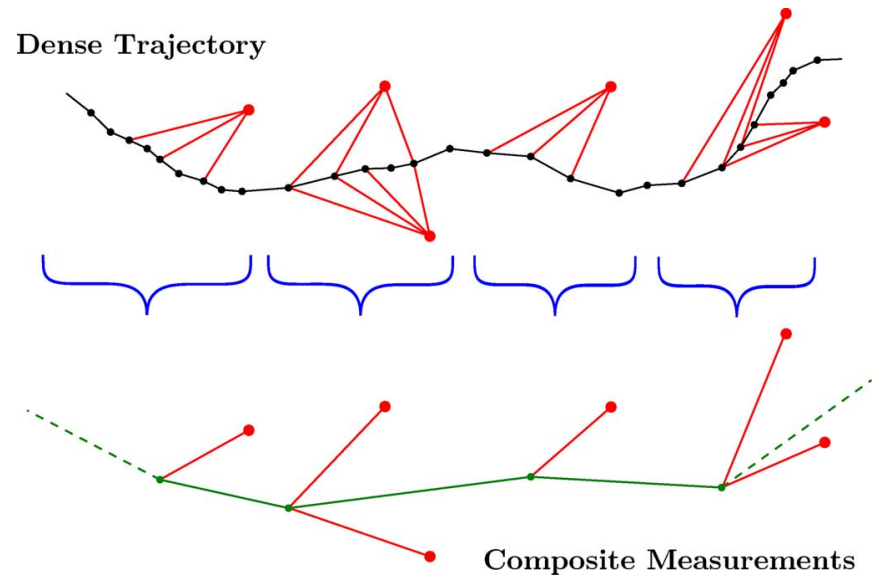

Fig. 8. The estimation problem can be viewed as a graph. As the robot explores, a pose (black) is added to the graph at each iteration while feature detections (red) are also added to produce a dense trajectory. This dense trajectory is very large so we periodically marginalize portions of the trajectory and the feature observations into composite measurements (green) at a much lower rate. The composite measurements are the input to the matching algorithm in Section V.

ilar in concept to key frames in Vision SLAM and is illustrated in Fig. 8.

The specific choice of $10 \mathrm{~s}$ is related to the speed of the AUV and the separation of the features. Also doing so at this rate (corresponding to one node every approximately 100 velocity measurements) keeps the optimization problem relatively small.

We time this marginalization step to occur after a feature has left the sonar FOV as this allows us to optimally estimate its relative location given all available information. This composite measurement is then added to a lower frequency higher level graph. This low-frequency graph is used as input to the prior map-matching algorithm in Section V. Meanwhile, the highfrequency graph begins to grow again by the insertion of newer constraints into $A_{i}$.

An alternative approach would be to maintain the dense trajectory of the robot pose at all times. This is the approach taken by iSAM [26], however, given the size of the resultant graph, we are not certain that such an approach would have been able to yield a computationally constant solution required for our low-powered embedded CPU onboard the AUV.

Additionally and unlike most land-based systems, the underwater domain is characterized by extended periods where the seabed is featureless for long distances. In that case, the resultant composite measurement is simply the relative trajectory of the distance traveled.

\section{B. Feature Tracking}

While Section IV-A explains how the graph of the trajectory and sonar observations is optimized and efficiently solved, we have not discussed the way in which sonar features are proposed.

The sonar detector passes point extractions to a feature nursery which maintains a vector of all recent detections. The nursery feature projects the detections into a local coordinate frame using the recent vehicle dead reckoning and uses a probabilistic distance threshold to associate them with one another. 

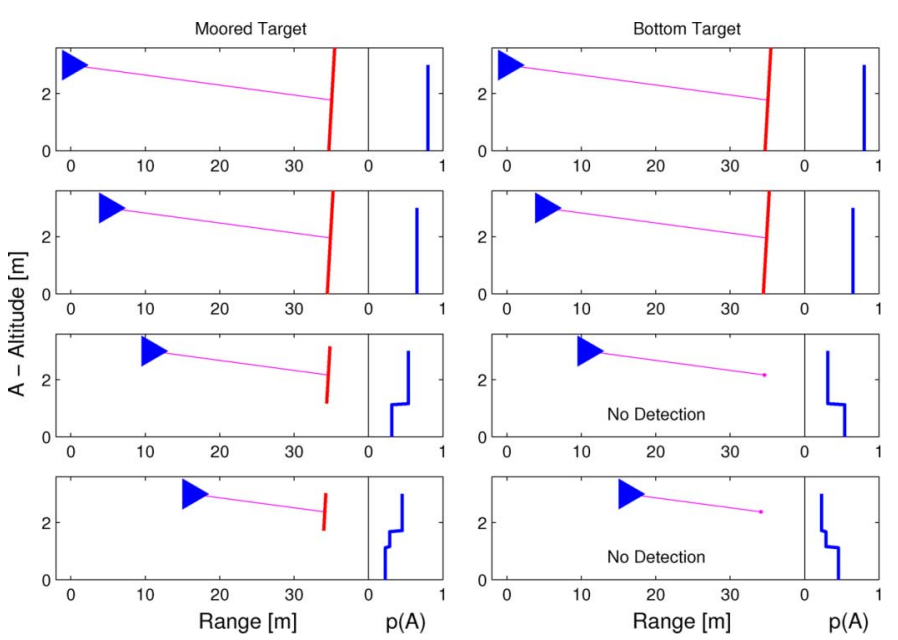

Fig. 9. A side-view pictorial illustrating convergence of feature height estimates for moored and bottom features - left and right columns respectively. On the left of each pictorial, an AUV (blue triangle), at 2.5-m altitude, approaches a feature (located at $35 \mathrm{~m}$ ) and senses it (as indicated by the magenta line) with a horizontal sonar facing $5^{\circ}$ down. The intensity of the feature detection infers a set of plausible off-beam heights (the red line indicates beam width). Multiple observations allow the altitude probability density function (pdf) $p(A)$ to converge to within a couple of meters. In the case of the bottom feature, no detections for the final two frames infer that the feature is below the main beam width. In real experiments, a feature could be detected as many as 30 times.

Should a sufficiently large number of detections be clustered together (approximately 7-8, dependent on the spread and intensity of detections), it is inferred that a consistent physical feature is present.

At this stage, the nursery feature is added to the square root smoother. All of the relative AUV-to-point constraints for that feature are then optimized, which results in improved estimation of the feature and the AUV trajectory. Subsequent point detections, inserted directly into the pose graph, result in an improved estimate via further square root smoothing.

This approach also estimates the height/altitude of the sonar feature, using the 2-D sonar intensities measured at each iteration. This amplitude distribution is non-Gaussian, and we model it instead as a cubic-normal distribution

$$
A(\phi)=\mathcal{N}\left(\left(\frac{\phi}{\phi_{\text {nom }}}\right)^{3} ; 0,1\right) A_{\text {meas }}
$$

where $A_{\text {meas }}$ is the actual measured intensity. This distribution is characterized as being relatively flat within the nominal lobe width $\phi_{\text {nom }}$ before dropping off quickly a few degrees beyond the lobe edge. This allows us to differentiate bottom from moored features. See Fig. 9 for a pictorial showing the height estimate of a feature position converging.

Finally, it should be noted that the input to this feature tracker are point features characterized only by their location and covariance, due to the low resolution of the sonar sensor. This makes it difficult to robustly associate nonconsecutive observations and, hence, to infer SLAM loop closures on the graph structure.

Additionally, clusters of nearby targets were prone to being mistakenly clustered into a single combined target. More careful estimation of target clusters is an interesting avenue of future work as well as being a possible avenue for successful loop closures.

\section{Global Estimation AND Map Matching}

Given the high level graph of the robot trajectory and observed feature locations, it still remains for the autonomous system to make a critical judgment of where it is relative to the a priori map and to decide if this relative match is certain enough to be declared convincingly. To do this, we maintain a set of match hypotheses in parallel. We compare them probabilistically so as to quantify the quality of the map match.

This comparison is implemented using a bank of estimators working in parallel, each tracking a different match hypothesis. The relative likelihood of one match hypothesis over another is computed using positive information (of prior features detected by the sonar) as well as negative information (of prior features that were expected but undetected by the sonar), and, in this way, matching can be done in a probabilistically rigorous manner. Negative information can be summarized as follows: if one expects to detect features along a proposed robot trajectory and these features were not seen, then the proposed trajectory is less likely.

The inclusion of this extra information is motivated by the regular structure of the feature field and the inability of positive information metrics to estimate the relative position of the AUV along these lines. The incorporation of negative information in this way is, to our knowledge, a novel contribution and was motivated by information not captured by algorithms such as joint compatibility branch and bound (JCBB) [27].

\section{A. Negative and Positive Scoring}

In SLAM, multihypothesis comparison can typically be reduced to a scoring algorithm of the relative probabilities of candidate solutions. Here we propose an algorithm for multihypothesis scoring which uses both positive as well as negative information: we name it the negative and positive scoring (NAPS) metric. An early version of this concept was introduced in [28].

We define NAPS $_{t}$ as the ratio of the probability of a map matching hypothesis $h_{i}$ when compared to a null hypothesis $h_{\text {null }}$, both conditioned (at each time step $t$ ) on the measurements $z_{1: t}$

$$
\begin{aligned}
\operatorname{NAPS}\left(h_{i}\right) & =\sum_{t=1}^{t} \operatorname{NAPS}_{t}\left(h_{i}\right) \\
\operatorname{NAPS}_{t}\left(h_{i}\right) & =\ln \left(\frac{p\left(h_{i} \mid z_{t}\right)}{p\left(h_{\mathrm{null}} \mid z_{t}\right)}\right) .
\end{aligned}
$$

We define a hypothesis $h$ as the combination of an estimate of the graph structure of the SLAM problem $x_{h}$ (the vehicle trajectory and all detected features) as well as all data association matches $\Omega$ of these features to map features in the prior map. The null hypothesis $h_{\text {null }}$ is a special version of this hypothesis in which no data associations exist and in which it is proposed that each detected feature is a new feature independent of the map. We use it as normalization for maps of growing size. 
Using Bayes' rule gives

$$
\operatorname{NAPS}_{t}\left(h_{i}\right)=\ln \left(\frac{p\left(z_{t} \mid h_{i}\right) p\left(h_{i}\right)}{p\left(z_{t} \mid h_{\text {null }}\right) p\left(h_{\text {null }}\right)}\right) .
$$

We split $p(z \mid h)$ into two terms representing both negative and positive information

$$
p\left(z_{t} \mid h\right)=\eta p\left(z_{t, \mathrm{pos}} \mid h\right) p\left(z_{t, \mathrm{neg}} \mid h\right) .
$$

Positive information is then defined, in the same way as for JCBB, as the likelihood of the measurements given the hypothesis

$$
\begin{aligned}
p\left(z_{t, \mathrm{pos}} \mid h\right) & =\eta_{z, \mathrm{pos}} e^{-\frac{1}{2}}\left(x_{h}-z_{t}\right)^{\mathrm{T}} \Sigma^{-1}\left(x_{h}-z_{t}\right) \\
& =\eta_{z, \mathrm{pos}} e^{-D_{h}}
\end{aligned}
$$

where $\Sigma$ represents the covariance, $\eta_{z \text {,pos }}$ is a normalization constant, and $D_{h}$ is the Mahalanobis distance.

The term $p(h)$ represents a prior probability of a particular map hypothesis being created by the robot which we propose is directly related to the number of features $N_{f}$ matched to the prior map and is given by

$$
p(h)=\eta_{x} e^{\lambda N_{f}}
$$

where $\eta_{x}$ is a normalization constant and $\lambda$ is a free parameter. $N_{f}$ is an integer between zero and the total number of features in the prior map. While this formulation does not take into account aspects such as a feature's measured visibility or other such specific terms, it does give us a measure of the confidence of a map match.

Combining these terms and canceling where possible gives the following expressions for NAPS as well as more common positive-only scoring (POS) metrics:

$$
\begin{aligned}
\operatorname{NAPS}_{t}(h) & =-D_{h}+\lambda N_{f}+C_{h, \text { neg }} \\
\operatorname{POS}_{t}(h) & =-D_{h}+\lambda N_{f} .
\end{aligned}
$$

This specifically indicates the contribution of negative information $C_{h, \text { neg }}$ that we believe has been neglected in typical multihypothesis scoring algorithms. POS algorithms (such as JCBB) implicitly assume $C_{h \text {, neg }}=0$ and do not account for it in scoring the hypotheses. Most approaches assume very-high $\lambda$ : essentially selecting the hypotheses that match the most total features and then ordering those by the Mahalanobis distance, as in the case of JCBB. A good overview of such algorithms is presented in [29] and [30].

\section{B. Evaluating Negative Information}

We define negative information as:

$$
\begin{aligned}
C_{h, \mathrm{neg}} & =\ln \left(\frac{p\left(z_{t, \mathrm{neg}} \mid h\right)}{p\left(z_{t, \mathrm{neg}} \mid h_{\text {null }}\right)}\right) \\
& =\ln \left(p\left(z_{t, \mathrm{neg}} \mid h\right)\right)-\ln \left(p\left(z_{t, \mathrm{neg}} \mid h_{\text {null }}\right)\right) .
\end{aligned}
$$

As each hypothesis NAPS score will eventually be compared to one another, the second term does not need to be calculated.
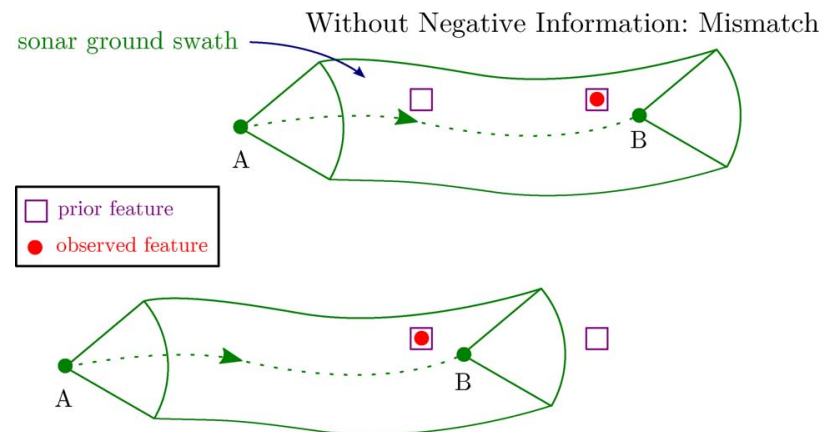

With Negative Information: Match corrected

Fig. 10. Illustration of the effect of NAPS. Consider the AUV trajectory from A to B with the sonar sensor footprint enclosed in green. If the AUV observes the red feature, how do we match its trajectory to the prior map (purple squares)? Using JCBB, the observed feature will be matched equally well to either prior feature. However, using negative information, NAPS indicates that the match in the lower figure is more likely. The upper figure is less likely because we would have expected to have observed both features, but only observed one.

For a particular hypothesis, consider an entire vehicle trajectory and the sonar footprint that it traced out (such as in Fig. 10). Also consider a prior map feature which is located within this footprint but was not detected. We wish to measure the number of times that this feature ought to have been detected, given that trajectory. Negative information is formed as the product of the probability of each undetected feature given the hypothesized vehicle trajectory

$$
\begin{aligned}
p\left(z_{t, \mathrm{neg}} \mid h\right) & =p\left(z_{t, \mathrm{neg}, f_{1}} \cap \ldots \cap z_{t, \mathrm{neg}, f_{n_{u}}} \mid h\right) \\
& =\prod_{f \in N_{u}} p\left(z_{t, \mathrm{neg}, f} \mid h\right) \\
& =\prod_{f \in N_{u}}\left(1-p\left(z_{t, \mathrm{pos}, f} \mid h\right)\right)
\end{aligned}
$$

where $s_{t}$ is the whole area sensed during measurement $z_{t}$, thus

$$
p\left(z_{t, \mathrm{pos}, f} \mid h\right)=\int_{p(f) \cap p\left(s_{t}\right)} v_{f} p(f) p\left(s_{t}\right) d A
$$

where $v_{f}$ is the visibility of feature $f$ and $p(f)$ is the prior probability of that feature.

In words, the probability of not detecting each conditionally independent feature is the product of one minus the probability of detecting each feature, integrated across the intersection of the pdf of each feature and the pdf of the scanned sensor area. This formulation is subject to the following assumptions: 1) the sensor occlusion model is well defined and accurate; 2 ) all features are static; 3) feature detections are independent; and 4) feature visibility can be accurately modeled. This calculation, often intractable due to complicated integration limits, theoretically defines the probability of a set of negative measurements $z_{t, \text { neg }}$ given sensed area $s_{t}$.

We approximate this measure using a grid-based approach in discrete cells around each composite measurement. The approach used an estimate of the total number of scans taken within a particular cell $s_{c}$. It is specified that to prevent spurious measurements from becoming incorrect features, there exists 
some number of positive detections $n_{d}$ associated to the same particular position (as discussed in Section IV-B). This allows computation of the $p\left(z_{t, \text { neg }} \mid h\right)$ term for each feature $f$ via the following binomial distribution:

$$
p\left(z_{t, \text { neg }, f} \mid h\right)=\sum_{j=0}^{n_{d}-1}\left(\begin{array}{c}
s_{c} \\
j
\end{array}\right) v_{f}^{(j)}\left(1-v_{f}\right)^{\left(s_{c}-j\right)} .
$$

These contributions are combined across all features predicted to lie in a mapped region via (23) to give $p\left(z_{t, \text { neg }} \mid h\right)$.

As the location of a feature is not exactly known, we assume a Gaussian distribution and use a Gaussian weighting $w_{c, f}$ of feature $f$ in cell $c$ within a $95 \%$ confidence ellipse (for computational reasons), which is summed to give an estimate of the number of scans of feature $f$ using

$$
l_{f}=\sum_{c \in \text { ellipse }} w_{c, f} s_{c} .
$$

This estimate is then used to calculate both the negative information contribution and the online estimate of feature visibility, via

$$
v_{f}=\frac{d_{f}}{l_{f}}
$$

where $d_{f}$ is simply the number of times feature $f$ is detected (on that specific pass), according to data association in the current hypothesis.

In terms of implementation, this approach requires the simple summation across each sonar scan. For the small number of features detected in this type of mission and the small number of hypotheses, this extensive summation is tractable for our system in real time. The result of the metric is a positive value which scores a particular hypothesis more likely when its observations do not contradict the prior map.

In particular, combining negative information with the other (positive-only) metrics in (19) allowed us to disambiguate similar locations along a row of otherwise indistinguishable features, as indicated in Fig. 10.

While the AUV operated in the field, this metric is evaluated for each hypothesis. The vehicle controls itself off of the most likely hypothesis, giving heading, speed, and depth commands to the low level vehicle controller so as to travel to a set of preprogrammed way points in the field. When the map match metric for a particular hypothesis exceeds a threshold, it is decided that the AUV is matched to the prior map and switches to a final target capture mode.

\section{Servoing to the FOI}

Having decided upon a confident matching to the prior map, the vehicle then homes to the estimated location of the FOI. When it approaches this location, the FOI should be observed in the sonar imagery. The mission controller then transitions to direct control using the local sonar detections and a proportional-integral-derivative (PID) controller on heading, which we call sonar servoing. It opens a pair of tines with a tip separation of approximately $1 \mathrm{~m}$ and drives onto the mooring line of
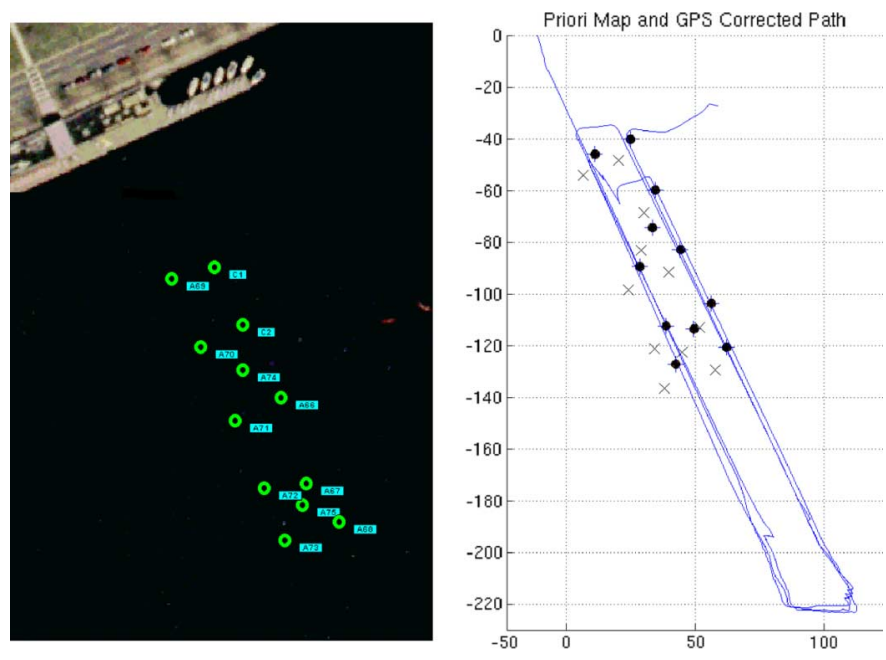

Fig. 11. Feature layout and vehicle path for an initial experiment (November 2006), demonstrating the basic capabilities of feature detection, local map construction, and global map matching running in real time onboard the Ranger unmanned underwater vehicle (UUV). Terminal homing to the feature of interest was added in subsequent experiments. Units are in meters.

the FOI. Detecting the abrupt change in velocity due to the line, the tine controller will clasp around the FOI ending the mission.

\section{FIELD EXPERIMENTS}

The system has undergone extensive testing and evolution over a five-year period. Starting in November 2006, we have conducted approximately 14 sea trials, each lasting two to three weeks. Our experiments began in fairly benign environments, using highly reflective moored objected as features, and progressed to more challenging conditions such as natural sea bottom features and strong currents. After each trial, we have refined and improved the system. In the following, we summarize the progress of the development of the algorithms and the vehicle platform.

Typically, the ingress point and direction to the field were varied for each mission while the choice of FOI was taken at random just before placing the AUV in the water. After reaching the field, the vehicle typically traveled along the rows of features indicated in Fig. 2. A typical mission visualization is presented in Fig. 12. This was so as to keep the number of map match hypotheses low (to about $4-5$ ). The typical mission duration was 15-25 min, although the mission planner could be programmed to repeat the mission if the AUV failed to find the feature field. During a successful mission, the AUV spent in the region of 4-5 min actually in the feature field. In all these missions, a $45^{\circ}$ sonar sensor was used. The typical water depth was $15 \mathrm{~m}$.

Detailed comparison of mission parameters is difficult because the effect of the vehicle's control decisions is that different paths (and observations) follow. For this reason, this section describes the progression of our core map matching algorithm.

1) Charles River and Boston Harbor, MA, USA (2006 and 2007): A series of initial experiments were carried out to establish the basic operation of the system in easier conditions. An overview of those missions is shown in Fig. 11.

2) St. Andrews Bay, FL, USA (June 2007): The NAPS and JCBB criteria were alternately used over 18 trials on a field of 
TABLE I

SElected Results for Homing EXPERIMENTS IN DifFERENT CONDITIONS, With AND Without Use OF NAPS

\begin{tabular}{|c|c|c|c|c|}
\hline Match Criteria & No. of Runs & Successes & Frequency & Significance \\
\hline \multicolumn{5}{|c|}{ Bright Features - June 2007} \\
\hline NAPS & 9 & 6 & $67 \%$ & $17 \%$ \\
\hline JCBB & 9 & 3 & $33 \%$ & $17 \%$ \\
\hline NAPS - JCBB (difference) & & & $33 \%$ & $24 \%$ \\
\hline NAPS \& Multihypothesis & 18 & 14 & $78 \%$ & $10 \%$ \\
\hline \multicolumn{5}{|c|}{ Normal Features - June 2008} \\
\hline NAPS Multihypothesis & 9 & 3 & $33 \%$ & $17 \%$ \\
\hline \multicolumn{5}{|c|}{ Normal Features, Low Currents - June 2009} \\
\hline NAPS Multihypothesis & 26 & 17 & $65 \%$ & $10 \%$ \\
\hline \multicolumn{5}{|c|}{ Normal Features, High Currents - June 2010} \\
\hline NAPS Multihypothesis & 42 & 13 & $31 \%$ & $7 \%$ \\
\hline \multicolumn{5}{|c|}{ As above, having reached the field - June 2010} \\
\hline NAPS Multihypothesis & 18 & 13 & $72 \%$ & $11 \%$ \\
\hline
\end{tabular}

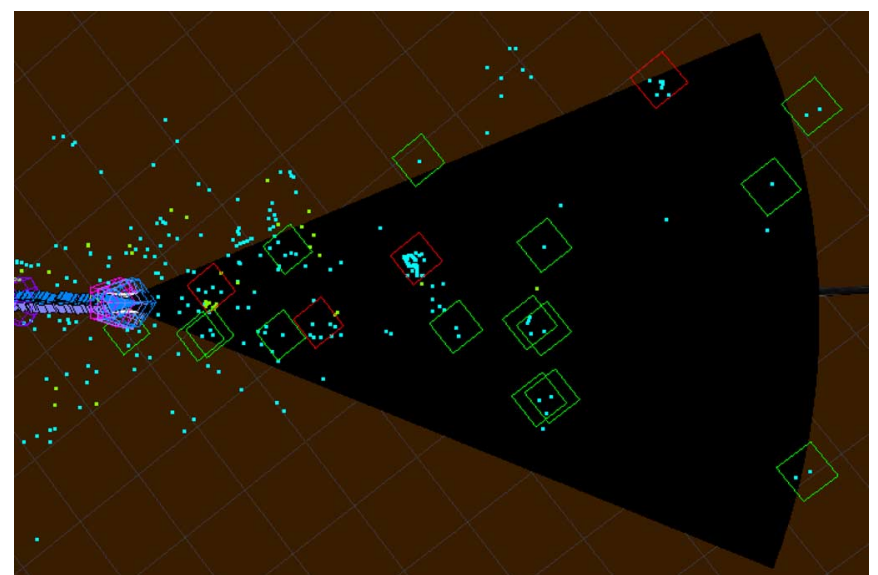

Fig. 12. Screenshot of the data viewer for an experiment performed in Monterey, CA, USA. Cyan dots are raw sonar detections, which are clustered as nursery features green squares. When justified, clustered nursery features are promoted to become FBN features, shown as red squares, and matched against the prior map.

strongly reflective moored features. The JCBB implementation uses a threshold on the Mahalanobis distance for multiple pair matching and chooses the most compatible pairs. The results of this live test and selected other tests are summarized in Table I.

In addition to the frequency of success, we have also presented the unbiased significance of these trials, which we calculated as

$$
S=\sqrt{\frac{n_{s}\left(n-n_{s}\right)}{(n-1) n^{2}}}
$$

where $n$ is the number of trials and $n_{s}$ is the number of successful trials. A lower value indicates a more confident result. We believe that the results from this trial demonstrate that the NAPS outperforms the simpler JCBB matching criteria for our application.

3) Narragansett Bay, RI, USA (June 2008): Using the data from June 2007, significant improvements to our sonar processing algorithms allowed for improved detection of

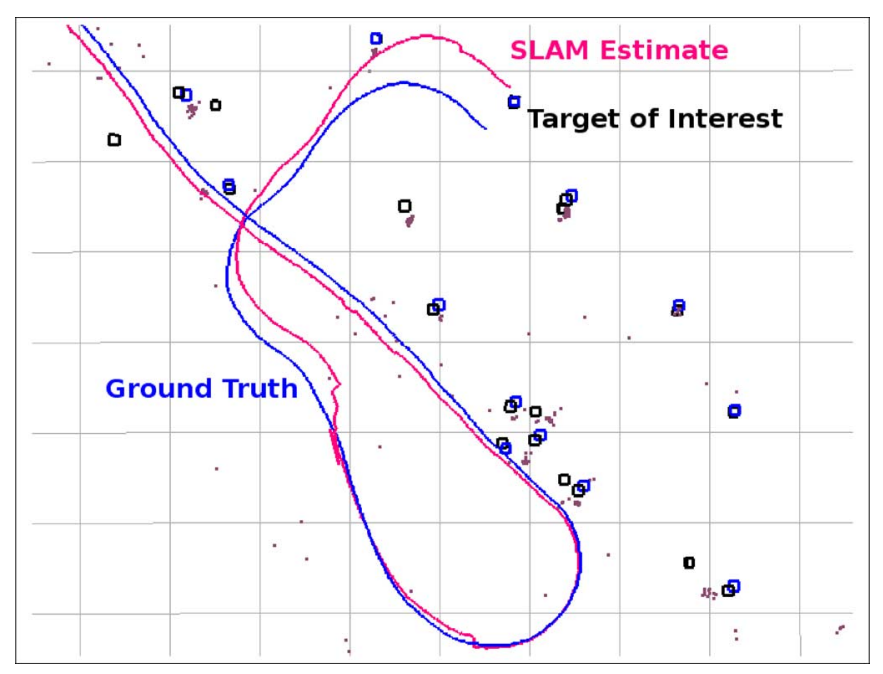

Fig. 13. A top-down overview of a successful mission using the REMUS 100 vehicle. The vehicle approached from the northwest and extracted feature points (purple dots). Using these points and the prior map (blue squares), the SLAM map (black squares) and the vehicle trajectory estimate (magenta line) were formed. Having matched against the map the vehicle homed to the FOI. The abrupt position changes are the result of new updates from the smoothing algorithm. The scale of the grid is $10 \mathrm{~m}$. It is important to note that the DVL-INS-enabled AUV would have failed to reacquire the FOI without using sonar as the map itself was only accurate to $5 \mathrm{~m}$ (blue line).

man-made and natural bottom features. This included the addition of an adaptive noise floor model discussed in Section III-A and a reimplementation in integer logic for increased efficiency. In addition, we began to test the control and attachment to the feature. The field for these tests consisted of various man-made and naturally occurring objects on the sea bottom as well as moored features. The bay had a significant tidal current comparable to the $0.5-\mathrm{m} / \mathrm{s}$ velocity of the vehicle, which gave us substantial dead-reckoning errors.

In the nine runs, we attached to the feature once and had two mechanical failures. In both cases, the tine mechanism broke upon hitting the target mooring line. Thus, the overall success rate of the sonar navigation system was 33\%. After these tests, the current model mentioned in Section II-B was developed. 
4) Stillwater Test Pond (March 2009): In this man-made test pond, we performed tests specifically on final control to the FOI and on the tine attachment mechanism. Only one feature was used and there were no currents, thus this experiment is not comparable to the others in terms of perceptive complexity. The pond was approximately $100 \mathrm{~m}$ wide at the location of the tests. The vehicle successfully latched onto the line in each of the 17 trials.

5) Gulf of Mexico, near Panama City, FL, USA (June 2009): The entire system was tested on a field of 12 bottom objects and three moored objects over a two-week period. These experiments tested an improved model for current estimation along with minor adjustments to the feature modeling. The current during this period was estimated as being $0.2 \mathrm{~m} / \mathrm{s}$ (using GPS surfaces). We had 17 successful feature attachments in 26 runs.

6) Gulf of Mexico (July 2010): The final set of experiments with the Ranger AUV were carried out. In this test, we observed much higher currents. These currents varied significantly from day to day but were estimated to be greater than the vehicle velocity (greater than $0.5 \mathrm{~m} / \mathrm{s}$ ) on certain days. In these conditions, the vehicle could not make any headway against the current when it found itself down-current from the feature field.

Presented in Table I are two different results for this experiment. One result gives the overall percent success when including all of the 42 runs carried out: $31 \%$. Filtering the runs to the 18 runs in which the AUV was able to enter the field (as defined by at least a single feature detection in the sonar) produced a success percentage of $72 \%$. We believe that this value is more in fitting with the performance of the SLAM algorithm and comparable to the previous year's results. Nonetheless, this demonstrates the limitation of this particular vehicle platform as well as ocean current estimation without a direct sensor.

7) Gulf of Mexico (May 2011): An additional series of tests was performed with the system running in real time onboard a REMUS AUV (without using its DVL). The motivation for porting the software to the REMUS was to be able to perform tests in a wider range of currents, and to compare trajectories estimated by the vehicle against the trajectory estimated by the REMUS's integrated Doppler INS. While further field tests are required to fully evaluate the system's performance, we were able to achieve a number of successful target reacquisitions, thereby demonstrating that the FBN approach is applicable to multiple types of AUVs. The results for a typical successful reacquisition mission with this vehicle are shown in Fig. 13.

\section{CONCLUSION AND Discussion}

This paper has developed a feature reacquisition system for small low-cost AUVs, based on FLS-based SLAM and demonstrated its performance in challenging and realistic field conditions. Our results indicate that when the AUV correctly matches to the prior feature map, it is regularly able to revisit a designated FOI. This was demonstrated by reattachment, using a pair of mechanical tines.

The main failure mode of the algorithm is failing to enter the feature field, due to disturbances that exceed the vehicle's control authority. For small-to-moderate ocean currents we developed an online current estimation procedure which allows the vehicle to avoid being driven off course during the initial vehicle dive. However, it should be noted that failure to successfully enter the field is not in itself a system failure as the vehicle could surface for a GPS fix before trying again. Nonetheless, room exists to improve the current estimation procedure described in Section II-B.

Unsurprisingly, in currents of more than $50 \%-70 \%$ of the vehicle's velocity, successful performance was limited. This presented an engineering limitation for this technology. As mentioned in Section II, our most recent work has been focused on transferring this project to an AUV with a greater velocity.

Given the generic appearance of individual sonar features, robust loop closure is difficult in this domain. However, with larger FOV sonars and denser feature fields, it may be possible to implement dense feature matching where more than a single feature are observed. This approach could well provide a single distinct loop closure constraint.

While more research is necessary to understand the many variables that can affect the system performance, such as the density and complexity of environmental features, the project has shown the viability of the FBN concept for feature reacquisition with low-cost vehicles.

\section{ACKNOWLEDGMENT}

The authors would like to thank their collaborators at iRobot, Blueview, Naval Postgraduate School, SeeByte Ltd., and Naval Surface Warfare Center Panama City for their assistance in this project, especially D. Horner, D. Kucik, E. Matson, B. Schulz, P. Carroll, and S. Reed.

\section{REFERENCES}

[1] D. B. Kilfoyle and A. B. Baggeroer, "The current state-of-the-art in underwater acoustic telemetry," IEEE J. Ocean. Eng., vol. 25, no. 1, pp. 4-27, Jan. 2000.

[2] L. Whitcomb, D. Yoerger, H. Singh, and D. Mindell, "Towards precision robotic maneuvering, survey, and manipulation in unstructured undersea environments," in Proc. Int. Symp. Robot. Res., 1998, vol. 8, pp. $45-54$

[3] J. J. Leonard, A. A. Bennett, C. M. Smith, and H. J. S. Feder, "Autonomous underwater vehicle navigation," Massachusetts Inst. Technol. (MIT), Cambridge, MA, USA, Tech. Rep. Mar. Robot. Lab. Tech. Memo. 98-1, 1998.

[4] H. Durrant-Whyte and T. Bailey, "Simultaneous localisation and mapping (SLAM): Part I,” Robot. Autom. Mag., vol. 13, no. 2, pp. 99-110, Jun. 2006

[5] B. Schulz, R. Hughes, R. Matson, R. Moody, and B. Hobson, "The development of a free-swimming UUV for mine neutralization," in Proc. IEEE/MTS OCEANS Conf. Exhibit., 2005, pp. 1443-1447.

[6] J. Long, J. Schumacher, N. Livingston, and M. Kemp, "Four flippers or two? tetrapodal swimming with an aquatic robot," Bioinspirat. Biomimet., vol. 1, pp. 20-29, 2006.

[7] J. C. Kinsey, R. M. Eustice, and L. L. Whitcomb, "A survey of underwater vehicle navigation: Recent advances and new challenges," in Proc. IFAC Conf. Manoeuver. Control Marine Craft, Lisbon, Portugal, Sep. 2006, DOI: 10.1.1.123.9809, invited paper.

[8] D. Meduna, "Terrain relative navigation for sensor-limited systems with application to underwater vehicles," Ph.D. dissertation, Dept. Aeronaut. Astronaut., Stanford Univ., Stanford, CA, USA, 2011.

[9] B. Cushman-Roisin and J. Beckers, Introduction to Geophysical Fluid Dynamics. New York, NY, USA: Academic, 2011, ch. 3-4, pp. 77-130.

[10] R. Thompson and W. Zehner, "Frequency-steered acoustic beam forming system and process," U.S. Patent $5923617,1999$.

[11] S. Negahdaripour, P. Firoozfam, and P. Sabzmeydani, "On processing and registration of forward-scan acoustic video imagery," in Proc. 2nd Can. Conf. Comput. Robot Vis., 2005, pp. 452-459. 
[12] S. Williams, G. Dissanayake, and H. Durrant-Whyte, "Towards terrainaided navigation for underwater robotics," Adv. Robot., vol. 15, pp. 533-550, 2001.

[13] H. Johannsson, M. Kaess, B. Englot, F. Hover, and J. Leonard, "Imaging sonar-aided navigation for autonomous underwater harbor surveillance," in Proc. IEEE/RSJ Int. Conf. Intell. Robots Syst., Taipei, Taiwan, Oct. 2010, pp. 4396-4403.

[14] I. T. Ruiz, S. de Raucourt, Y. Petillot, and D. Lane, "Concurrent mapping and localization using sidescan sonar,” IEEE J. Ocean. Eng., vol. 29, no. 2, pp. 442-456, Apr. 2004.

[15] N. Fairfield, A. G. Kantor, and W. D. , "Real-time SLAM with octree evidence grids for exploration in underwater tunnels," J. Field Robot., vol. 24, pp. 3-21, 2007.

[16] Y. Petillot, I. T. Ruiz, and D. M. Lane, "Underwater vehicle obstacle avoidance and path planning using a multi-beam forward looking sonar," IEEE J. Ocean. Eng., vol. 26, no. 2, pp. 240-251, Apr. 2001.

[17] D. E. Clark and J. Bell, "Bayesian multiple target tracking in forward scan sonar images using the PHD filter," Inst. Electr. Eng. Proc.-Radar Sonar Navig., vol. 152, pp. 327-334, 2005.

[18] M. G. Dissanayake, P. Newman, S. Clark, H. Durrant-Whyte, and M. Csorba, "A solution to the simultaneous localization and map building (SLAM) problem," IEEE Trans. Robot., vol. 17, no. 3, pp. 229-241, Jul. 2001.

[19] P. Newman, J. J. Leonard, and R. Rikoski, "Towards constant-time SLAM on an autonomous underwater vehicle using synthetic aperture sonar," Int. J. Robot. Res., vol. 1, pp. 309-420, 2003.

[20] M. Montemerlo, S. Thrun, D. Roller, and B. Wegbreit, "FastSLAM 2.0: An improved particle filtering algorithm for simultaneous localization and mapping that provably converges," in Proc. Int. Joint Conf. Artif. Intell., 2003, pp. 1151-1156.

[21] G. Grisetti, C. Stachniss, and W. Burgard, "Improved techniques for grid mapping with Rao-Blackwellized particle filters," IEEE Trans. Robot., vol. 23, no. 1, pp. 34-46, Feb. 2007.

[22] S. Julier and J. Uhlmann, "A counter example to the theory of simultaneous localization and map building," in Proc. IEEE Int. Conf. Robot. Autom., 2001, vol. 4, pp. 4238-4243.

[23] F. Dellaert and M. Kaess, "Square root SAM: Simultaneous location and mapping via square root information smoothing," Int. J. Robot. Res., vol. 25, no. 12, pp. 1181-1204, Dec. 2006.

[24] F. Dellaert and M. Kaess, "Square Root SAM: Simultaneous localization and mapping via square root information smoothing," Int. J. Robot. Res., vol. 25, no. 12, pp. 1181-1203, Dec. 2006.

[25] J. Folkesson and J. Leonard, "Autonomy through SLAM for an underwater robot," in Robotics Research: The 14th International Symposium ISRR, ser. Springer Tracts in Advanced Robotics. New York, NY, USA: Springer-Verlag, 2011, vol. 70, pp. 55-70.

[26] M. Kaess, A. Ranganathan, and F. Dellaert, "iSAM: Incremental smoothing and mapping," IEEE Trans. Robot., vol. 24, no. 6, pp. 1365-1378, Dec. 2008

[27] J. Neira and J. D. Tardos, "Data association in stochastic mapping using the joint compatibility test," IEEE Trans. Robot., vol. 17, no. 6, pp. 890-897, Dec. 2001.

[28] J. Folkesson and J. Leonard, "Autonomy through SLAM for an underwater robot," in Proc. Int. Symp. Robot. Res., 2009.

[29] J. Neira, J. Tardos, and J. Castellanos, "Linear time vehicle relocation in SLAM," in Proc. IEEE Int. Conf. Robot. Autom., Sep. 2003, pp. 427-433.

[30] L. Paz, J. Guivant, J. Tardós, and J. Neira, "Data association in O(n) for divide and conquer SLAM," in Proc. Robot., Sci. Syst. (RSS), Atlanta, GA, USA, Jun. 2007, pp. 281-288.

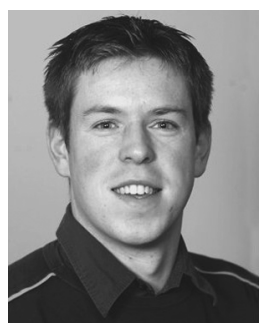

Maurice F. Fallon received the B.Eng. degree in electronic and electrical engineering from University College Dublin (UCD), Dublin, Ireland and the $\mathrm{Ph} . \mathrm{D}$. degree in information engineering from the University of Cambridge, Cambridge, U.K.

$\mathrm{He}$ is a Research Scientist in the Computer Science and Artificial Intelligence Laboratory (CSAIL), Massachusetts Institute of Technology (MIT), Cambridge, MA, USA. His areas of research interest include sequential Monte Carlo methods, probabilistic mobile robotics including simultaneous localization and mapping (SLAM) and perception. More recently, his research has involved mobile perception for mobile platforms including for the visually impaired and for walking robots.

Dr. Fallon received the UCD President's Prize in 2007 and the Microsoft European Ph.D. Scholarship in 2008. He is the perception lead of MIT's DARPA Robotics Challenge team.

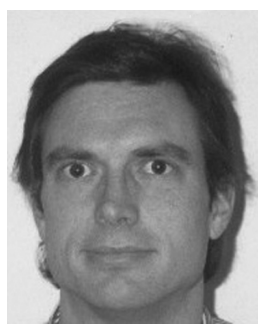

John Folkesson received the B.A. degree in physics from the City University of New York, Queens College, Kew Gardens Hills, Queens, NY, USA, in 1983 and the M.Sc. degree in computer science and the $\mathrm{Ph} . \mathrm{D}$. degree in autonomous systems from the Royal Institute of Technology (KTH), Stockholm, Sweden, in 2001 and 2006, respectively, where he became a Docent in Robotics in 2012.

$\mathrm{He}$ is a Research Scientist at the Center for Autonomous Systems, KTH. He was a Postdoctoral Fellow at the Department of Mechanical Engineering, Massachusetts Institute of Technology (MIT), Cambridge, MA, USA (2006-2007) and continued there as a research scientist until 2010. Since 2010 , he has been at KTH. His research has covered robotic systems in indoor, outdoor, and underwater environments. He has developed graphical methods for Bayesian inference in robot navigation. Currently, his research has widened to include spatial reasoning and long-term learning of spatial knowledge.

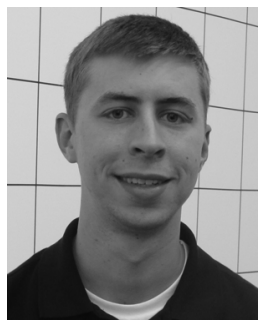

Hunter McClelland received the B.S. degree from the Tennessee Technological University, Cookeville, TN, USA and the M.S. degree from the Massachusetts Institute of Technology (MIT), Cambridge, MA, USA. He is currently working toward the Ph.D. degree in mechanical engineering at the Virginia Polytechnic Institute of Technology and State University (Virginia Tech), Blacksburg, VA, USA.

His research interest is in robotic navigation and control, specializing in algorithms for dynamics, optimization, trajectory generation, navigation, and simultaneous localization and mapping.

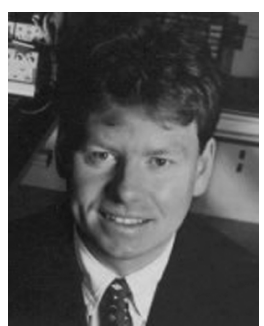

John J. Leonard is a Professor of Mechanical and Ocean Engineering at the Massachusetts Institute of Technology (MIT), Cambridge, MA, USA and a member of the MIT Computer Science and Artificial Intelligence Laboratory. $\mathrm{He}$ is Co-Director of the Ford-MIT Alliance and Area Head for Ocean Science and Engineering in the MIT Department of Mechanical Engineering. His research addresses the problems of navigation and mapping for autonomous mobile robots. With his students and collaborators, he has developed several state-of-the-art robot navigation and mapping systems for robots operating in underwater and terrestrial environments. He was team leader for MIT's DARPA Urban Challenge team, which was one of 11 teams to qualify for the Urban Challenge final event and one of six teams to complete the race.

Prof. Leonard has served as an Associate Editor of the IEEE JOURNAL OF OCEANIC ENGINEERING and of the IEEE TRANSACTIONS ON ROBOTICS AND Automation. He was one of three guest editors for the 2008 IEEE TRANSACTIONS ON RoBotics Special Issue on Visual SLAM. He is the recipient of a Thouron Award (1987), a National Science Foundation (NSF) Career Award (1998), a Science Foundation Ireland E.T.S. Walton Visitor Award (2004), the Best Paper Award at ACM SenSys in 2004, and the King-Sun Fu Memorial Best Transactions on Robotics Paper Award in 2006. 